Original Article

\title{
Is hypertension a risk factor for poor balance control in elderly adults?
}

\author{
Serap Acar ${ }^{1)^{*}}$, İlkşan Demírbüken ${ }^{2)}$, Candan Algun $^{3)}$, Mehtap Malkoç $^{1)}$, Nil Tekin ${ }^{4}$ ) \\ 1) School of Physical Therapy and Rehabilitation, Dokuz Eylul University: 35340 Inciralti, İmir, \\ Turkey \\ 2) Department of Physiotherapy and Rehabilitation, Faculty of Health Sciences, Marmara University, \\ Turkey \\ 3) Department of Physical Therapy and Rehabilitation, Faculty of Medical Sciences, Medipol \\ University, Turkey \\ 4) SGK Narlidere Geriatric Home Care Center, Turkey
}

\begin{abstract}
Purpose] The aim of this study was to investigate whether hypertension negatively affects the postural balance control of elderly adults under different sensory conditions. [Subjects and Methods] Fifty-four healthy elderly adults who were residents in a Geriatric Home Care Center were recruited for this study. Height, weight, body mass index and age of the volunteers were recorded. After applying the exclusion criteria, the final study group included 16 hypertensive (HT) and the control group included 10 non-hypertensive (Non-HT) healthy elderly adults. To evaluate postural balance control objectively, the modified Clinical Test of Sensory Interaction on Balance (modified CTSIB) test was performed under four different conditions: 1) eyes open on a stable surface; 2) eyes closed on a stable surface; 3 ) eyes open on an unstable surface; and 4) eyes closed on an unstable surface. [Results] The postural balance scores (center of gravity sway) of the HT group were slightly higher than those of the Non-HT group under conditions 1 (HT group $=0.3 \% \mathrm{sec}$, Non-HT group $=0.2 \% \mathrm{sec}$ ), 2 (HT group $=0.8 \% \mathrm{sec}$, NonHT group $=0.4 \% \mathrm{sec})$ and $4(\mathrm{HT}$ group $=4.5 \% \mathrm{sec}$, Non-HT group $=3.5 \% \mathrm{sec})$, but no statistically significant differences were found between the HT and Non-HT groups under any sensory condition. [Conclusion] The result of this study indicate that controlled hypertension in elderly adults is not a cause of worse balance performance than controls on stable or unstable surfaces with the eyes open or closed.

Key words: Hypertension, Postural balance, Posture
\end{abstract}

(This article was submitted Sep. 30, 2014, and was accepted Nov. 7, 2014)

\section{INTRODUCTION}

Maintenance of postural balance is one of the most important abilities required for the prevention of falls in the elderly population. Unfortunately, with increasing age, sensory and motor control of postural balance is often affected ${ }^{1)}$.

Several studies have been performed to determine risk factors of falls and their relation to balance control in elderly adults. Health problems common in the elderly such as polypharmacy, impaired cognition, pain, and urinary incontinence have consistently been found to increase fall risk through their effects on the sensory and motor components of postural balance ${ }^{2,3)}$.

The prevalence of hypertension also increases with age $e^{4,5)}$ and hypertension is frequently accompanied by symptoms such as headache, blurring of vision, tachycardia, thoracic

\section{*Corresponding author. Serap Acar (E-mail: acarserap2@} gmail.com)

(ㄱ2015 The Society of Physical Therapy Science. Published by IPEC Inc. This is an open-access article distributed under the terms of the Creative Commons Attribution Non-Commercial No Derivatives (by-ncnd) License $<$ http://creativecommons.org/licenses/by-nc-nd/3.0/> . pain, shortness of breath, weakness of limbs and swollen ankles ${ }^{6,7)}$. These symptoms are considered to be factors influencing anatomical or functional alterations affecting postural balance ${ }^{8)}$.

Hypertension can also negatively affect balance by damaging the large arteries and decreasing microcirculation in specific functional areas ${ }^{8,9)}$. This would result in impaired stimulus reception from the peripheral structures and environment, thereby decreasing the ability to maintain a stable posture ${ }^{10)}$. Furthermore, the rapid changes in blood pressure seen in hypertension and the subsequent sudden reductions in blood flow can also interfere with the mechanisms for controlling postural balance ${ }^{8)}$.

Age and abnormalities in blood pressure homeostasis may predispose individuals to orthostatic hypotension, a very common condition among older adults. Reduced arterial compliance has been shown to be associated with a reduction in baroreflex sensitivity, a key mechanism of short-term blood pressure regulation. A recent, relatively small, study found that arterial stiffness of the upper limbs was significantly higher in patients with orthostatic hypotension and a history of falls ${ }^{11)}$.

Hormonal reactions in response to orthostasis include changes in the renin-angiotensin-aldosterone system, pro- 
motion of sodium and water retention leading to increase of blood volume, and the release of other peptides and active neuroamines such as vasopressin and epinephrine, whose effects are seen within minutes of orthostasis. Vasopressin release from the posterior pituitary in response to minimal changes in blood volume is initiated by stimulation of the atrial stretch receptors and arterial baroreceptors ${ }^{12)}$.

It has been established that vision is the primary sensory system used in balance. Peripheral vision is sensitive to movement and is thought to dominate both perception of self-motion and postural control. Gaerlen et al. found that when subjects clesed their eyes, trunk sway increased threefold, supporting the notion that vision plays a predominant role in balance. Therefore, visual input is more critical for the maintenance of balance than stimuli from the vestibular and proprioceptive systems, and the visual system must be assessed when testing balance ${ }^{15}$.

Hypertension may also result in impaired visual ability due to retinopathy $\left.{ }^{13}, 14\right)$. Therefore, most components of postural balance control can be influenced by hypertension.

Several studies have been conducted of the balance control of hypertensive elderly adults, but they have reported conflicting results. Cho et al. stated that balance control or normal postural control is essential for mobility as well as stability during functional activities since deficits in balance can lead to falls by the elderly ${ }^{16}$. Thus, better understanding of the relationship between hypertension and postural balance control in elderly adults may help to better determine the fall risks of this population.

The aim of this study was to investigate the hypothesis that hypertension affects postural balance control in elderly adults unfavorably under different sensory conditions.

\section{SUBJECTS AND METHODS}

\section{Subjects}

Fifty-four healthy elderly adults who lived in a Geriatric Home Care Center were recruited for this study. All residents who volunteered and met the following inclusion criteria were included: $\geq 75$ years old; able to perform self-care activities of daily living without difficulties or need of help; and able to walk independently without an assistive device. Patients were excluded from the study if they had impaired cognitive status (a score of $<24$ in the Mini Mental State Examination), psychiatric disorders requiring drug treatment, a diagnosis of diabetes mellitus, vestibular disorders, previous lower limb surgery, active cancer requiring chemotherapy, neurological disorders influencing postural balance control, or visual disorders (not able to see further than 4 meters). Subjects who were using four or more medications were also excluded to eliminate the negative effects of polypharmacy on postural balance control.

Study participants signed an informed consent form in accordance with the procedures approved by the Local Ethical Committee. After recruitment of the 54 subjects, the study group included 16 hypertensive (HT) and 10 nonhypertensive (Non-HT) healthy elderly adults.

All HT subjects were diagnosed with hypertension by a cardiologist and were receiving anti-hypertensive therapy. At the start of the study blood pressure measurements were made to identify the clinical characteristics of the subjects. The subjects took their anti-hypertensive medication on the test day.

Medical history (Mini Mental State Examination scores, Basic Activities of Daily Living scores, diagnosis, drug treatment) were obtained from the medical reports of the Geriatric Home Care Center.

Prior to assessing postural balance control, the subjects' height, weight, body mass index and age were recorded. Systolic (SBP) and diastolic blood pressures (DBP) were measured three times and the mean values were recorded before the balance test. Hypertension was defined as the presence of SBP $>140 \mathrm{mmHg}$ and DBP $>90 \mathrm{mmHg}$. Volunteers were checked for balance-related symptoms such as vertigo or unsteadiness at the time of testing.

\section{Methods}

To objectively evaluate postural balance control, the modified Clinical Test of Sensory Interaction on Balance (modified CTSIB) test was performed on a NeuroCom Balance Master System (NeuroCom System Version 8.1.0, NeuroCom ${ }^{\circledR}$ International Inc., USA). A force platform connected to a computer detects center of gravity (COG) sway during different tasks. During the measurements, subjects were barefoot and positioned on the platform facing the monitor. The subjects were instructed not to consume any caffeine or alcohol for 48 hours before the test. During the test, the subjects were asked to stand comfortably, relaxed and as upright as possible. Two physical therapists were present during each subject's testing. One administered the test, and the other stood nearby to help subjects feel secure and prevent falls during testing. All the tests were conducted in the early morning at the Movement Science Laboratory of the School of Physical Therapy and Rehabilitaiton Dokuz Eylul University. All the participants were transported by service car and rested before the tests.

Modified CTSIB were performed under four sensory conditions: eyes open on a firm surface; eyes closed on a firm surface; eyes open on an unstable surface; eyes closed on an unstable surface. Each condition was tested three times. Trial data consisted of COG sway velocity in degrees per second, with higher values indicating poorer postural balance control.

Statistical analyses were performed using SPSS for Windows, version 11.0. The data were analyzed using the MannWhithey $U$ test to reveal whether there was a significant difference between the sway velocities of the hypertensive and non-hypertensive elderly adults. Statistical significance was chosen as $\mathrm{p}<0.05$ for all analyses, and the data were expressed as mean $\pm \mathrm{SD}$. Additionally, retrospective power analyses were conducted on the study population.

\section{RESULTS}

The demographic and clinical characteristics of the HT and Non-HT subjects are shown in Table 1. No statistically significant differences were found between the two groups in age, height, weight, BMI, SBP or DBP ( $p>0.05)$.

The postural balance values (COG sway) of the HT group were slightly higher than those of the Non-HT group under 
Table 1. Demographic and clinical characteristics of the HT and Non-HT subjects

\begin{tabular}{lcc}
\hline & HT Group & Non-HT Group \\
\cline { 2 - 3 } & Mean \pm SD & Mean \pm SD \\
\hline Age (years) & $80.5 \pm 2.8$ & $79.8 \pm 3.8$ \\
Height $(\mathrm{cm})$ & $159.6 \pm 9.3$ & $158.7 \pm 11.0$ \\
Body weight $(\mathrm{kg})$ & $71.7 \pm 12.1$ & $71.0 \pm 14.8$ \\
BMI $\left(\mathrm{kg} / \mathrm{m}^{2}\right)$ & $28.7 \pm 10.1$ & $29.6 \pm 9.2$ \\
SBP $(\mathrm{mmHg})$ & $135.0 \pm 21.0$ & $148 \pm 11.2$ \\
DBP $(\mathrm{mmHg})$ & $84.0 \pm 18.0$ & $85.0 \pm 13.0$ \\
\hline
\end{tabular}

HT and Non- HT: hypertensive and non-hypertensive, SD: standard deviation, BMI: body mass index, SBP and DBP: systolic and diastolic blood pressures

sensory condition 1 (HT group: $0.3 \%$ sec, Non-HT group: $0.2 \% \mathrm{sec}$ ), condition 2 (HT group: $0.8^{\circ} / \mathrm{sec}$, Non-HT group: $0.4 \% \mathrm{sec}$ ) and condition 4 (HT group: $4.5^{\circ} / \mathrm{sec}$, Non-HT group: $3.5 \% \mathrm{sec}$ ), but the differences were not statistically significant $(\mathrm{p}>0.05)$ (Table 2$)$.

Both groups had the same COG sway value under sensory condition $3(1.2 \% \mathrm{sec})$.

Unstable surface conditions worsened the postural balance stability of both the HT and Non-HT groups compared to firm surfaces. COG sway was greatest when subjects eyes were closed on the unstable surface (sensory condition 4).

For the retrospective power analysis, the power was estimated to be $100 \%$ with a $95 \%$ confidence interval for this study. The sample size was based on the number of the participants in similar studies.

\section{DISCUSSION}

The purpose of this study was to determine whether hypertension worsens the postural balance control of elderly adults. Contrary to our hypothesis, our findings show hypertension had no significant effect on the postural balance control of the elderly adults who participated in this study.

It has been reported in the literature that hypertensive subjects suffer from vertigo and dizziness, which are usually associated with poor balance control ${ }^{8}$. In addition to these symptoms, the systemic effects of hypertension on arteries and microcirculation may adversely affect postural balance centers in the central nervous system (CNS) (cerebellum and cochleo-vestibular system $)^{17,18)}$. Hausdorff et al. supported the hypothesis that hypertension negatively affects the balance control of elderly adults demonstrating that increased blood pressure not only led to cardiovascular disease, but also impacted balance, gait and fall risk $^{19)}$. It is worth noting that they used Tinnetti's performance-oriented assessment of mobility to measure postural balance and gait, which is a different methodology than that employed in the present study ${ }^{19)}$.

On the other hand, Abate et al. reported results consistent with our present study. They found no differences between balance tests of hypertensive and non-hypertensive elderly adults using posturographic measurements. However, they conducted only static tests on a stable force platform with
Table 2. Postural balance scores (COG sway velocities) of the HT and Non-HT groups

\begin{tabular}{lcc}
\hline & $\begin{array}{c}\text { HT Group } \\
\text { mean } \pm \text { SD }\end{array}$ & $\begin{array}{c}\text { Non-HT } \\
\text { Group } \\
\text { mean } \pm \text { SD }\end{array}$ \\
\hline Eyes open firm surface $(\% / s)$ & $0.3 \pm 0.1$ & $0.2 \pm 0.1$ \\
Eyes closed firm surface $(\% / \mathrm{s})$ & $0.8 \pm 1.3$ & $0.4 \pm 0.2$ \\
Eyes open unstable surface $(\% / \mathrm{s})$ & $1.2 \pm 1.3$ & $1.2 \pm 0.5$ \\
Eyes closed unstable surface $(\%)$ & $4.5 \pm 1.9$ & $3.5 \pm 1.7$ \\
\hline
\end{tabular}

HT: hypertensive, $\%$ s: degree $/$ second

the subjects' eyes open and did not measure the balance scores of hypertensive elderly adults under unstable conditions ${ }^{8)}$. The present study tested the balance ability of elderly subjects with their eyes both open and closed, and on stable and unstable surfaces. To our knowledge, there is only one other study that has compared balance ability under this range of conditions. Kim compared balance control under six different sensory conditions and found that elderly patients younger than 75 years old had significantly better postural balance with their eyes closed on a swaying surface than subjects over 75 years old ${ }^{20)}$. To the best of our knowledge, the present study is the first to investigate the relationship between hypertension and balance control in elderly adults under different sensory conditions.

The unimpaired balance control of elderly hypertensive adults in our study may be a result of effective medical management of their hypertension. The study participants were being treated under the supervision of their nurses at the Geriatric Home Care Center; therefore, these findings cannot be extended to community-dwelling elderly adults with hypertension ${ }^{18)}$. Compliance with proper usage of blood pressure medications is a well- known problem that is further compounded among older adults ${ }^{19)}$. Hajjar indicated that antihypertensive medication might improve postural blood pressure changes ${ }^{21)}$.

In postural blood pressure control, age-related declines in baroreflex sensitivity, cerebral blood flow, and renal sodium conservation threaten normal blood pressure regulation and cerebral perfusion. Common clinical conditions associated with abnormal blood pressure homeostasis and falls in the elderly include postural hypotension, postprandial hypotension, carotid sinus hypersensitivity, and cardiac arrhythmia, all of which are exacerbated by hypertension ${ }^{22}$.

However, postural balance control involves multiple systems besides the cardiovascular system. The evidence indicates that somatosensory, visual, vestibular, musculoskeletal, and central nervous system integration of inputs from the peripheral and motor systems declines with age ${ }^{23}$. This physiological deterioration may have had a more prominent effect on postural balance control than hypertension did in the study population, but these factors were not the focus of this study.

A limitation of this study is that the number of the participants was not sufficient to support the hypothesis. However, as this study was not planned as multicenter research, the number of the participants was limited by the population of the Geriatric Home Care Center. Future studies are planned 
to apply the same methods in multicenter research.

In conclusion, in this study elderly adults with controlled hypertension did not show worse balance performance than non-hypertensive elderly adults under stable or unstable sensory conditions with the eyes open or closed. Further research on postural balance control and hypertension is required to determine whether hypertension is a risk factor of impaired balance and falls in the elderly population.

\section{REFERENCES}

1) Sturnieks DL, St George R, Lord SR: Balance disorders in the elderly. Neurophysiol Clin, 2008, 38: 467-478. [Medline] [CrossRef]

2) Delbaere K, Close JC, Heim J, et al.: A multifactorial approach to understanding fall risk in older people. J Am Geriatr Soc, 2010, 58: 1679-1685. [Medline] [CrossRef]

3) Tinetti ME, Kumar C: The patient who falls: "It's always a trade-off", JAMA, 2010, 303: 258-266. [Medline] [CrossRef]

4) Zito M, Parati G, Omboni S, et al.: Effect of ageing on blood pressure variability. J Hypertens Suppl, 1991, 9: S328-S329. [Medline] [CrossRef]

5) Karavidas A, Lazaros G, Tsiachris D, et al.: Aging and the cardiovascular system. Hellenic J Cardiol, 2010, 51: 421-427. [Medline]

6) Fletcher AE, Bulpitt $\mathrm{CJ}$ : The measurement of quality of life in evaluating ACE inhibitors. J Hum Hypertens, 1990, 4: 45-49, discussion 49-50. [Medline]

7) Croog SH, Levine S, Testa MA, et al.: The effects of antihypertensive therapy on the quality of life. N Engl J Med, 1986, 314: 1657-1664. [Medline] [CrossRef]

8) Abate M, Di Iorio A, Pini B, et al.: Effects of hypertension on balance as sessed by computerized posturography in the elderly. Arch Gerontol Geriatr, 2009, 49: 113-117. [Medline] [CrossRef]

9) Hentschel F, Damian M, Krumm B, et al.: White matter lesions-age-adjusted values for cognitively healthy and demented subjects. Acta Neurol Scand, 2007, 115: 174-180. [Medline] [CrossRef]

10) Anile C, De Bonis P, Di Chirico A, et al.: Cerebral blood flow autoregulation during intracranial hypertension: a simple, purely hydraulic mechanism? Childs Nerv Syst, 2009, 25: 325-335, discussion 337-340. [Medline]
[CrossRef]

11) Mattace-Raso FU, van der Cammen TJ, Knetsch AM, et al.: Arterial stiffness as the candidate underlying mechanism for postural blood pressure changes and orthostatic hypotension in older adults: the Rotterdam Study. J Hypertens, 2006, 24: 339-344. [Medline] [CrossRef]

12) Perlmuter LC, Sarda G, Casavant V, et al.: A review of orthostatic blood pressure regulation and its association with mood and cognition. Clin Auton Res, 2012, 22: 99-107. [Medline] [CrossRef]

13) Polak BC, Meenken CI, Smulders YM: [Visual loss as a sign of hypertension]. Ned Tijdschr Geneeskd, 2009, 153: A379. [Medline]

14) Mensah A, Milea D, Jensen R, et al.: Persistent visual loss in malignant idiopathic intracranial hypertension. Acta Ophthalmol (Copenh), 2009, 87: 934-936. [Medline] [CrossRef]

15) Gaerlan MG, Alpert PT, Cross C, et al. Postural balance in young adults: the role of visual, vestibular and somatosensory systems. J American Acad Nurse Pract, 2012, 1-7.

16) Cho GH, Hwangbo G, Shin HS: The effects of virtual reality-based balance training on balance of the elderly. J Phys Ther Sci, 2014, 26: 615-617. [Medline] [CrossRef]

17) Pirodda A, Brandolini C, Carlo Modugno G: Hypotension associated with autonomic dysfunction: a possible cause of vertigo? Med Hypotheses, 2004, 63: 1086. [Medline] [CrossRef]

18) Borghi C, Modugno GC, Brandolini C, et al.: Is tinnitus useful in early detection of incoming heart decompensation? Med Hypotheses, 2006, 67: 437-439. [Medline] [CrossRef]

19) Hausdorff JM, Herman T, Baltadjieva R, et al.: Balance and gait in older adults with systemic hypertension. Am J Cardiol, 2003, 91: 643-645. [Medline] [CrossRef]

20) Kim YW: Correlation between the sensory organisation test and the functional reach test in balance evaluation of elderly individuals. J Phys Ther Sci, 2012, 24: 675-679. [CrossRef]

21) Hajjar I: Postural blood pressure changes and orthostatic hypotension in the elderly patient: impact of antihypertensive medications. Drugs Aging, 2005, 22: 55-68. [Medline] [CrossRef]

22) Lipsitz LA: Abnormalities in blood pressure homeostasis that contribute to falls in the elderly. Clin Geriatr Med, 1985, 1: 637-648. [Medline]

23) Fujita $T$, Nakamura $S$, Ohue $M$, et al.: Effect of age on body sway assessed by computerized posturography. J Bone Miner Metab, 2005, 23: 152-156. [Medline] [CrossRef] 